\title{
Caracterización del Lactante Sibilante Recurrente hospitalizado y Utilidad del Estudio Internacional de Sibilancias
}

\author{
Characterization of hospitalized Infants with recurrent wheezing and evaluation of \\ the International Study of Wheezing
}

\section{Mirna Elizabeth Diaz Romero,* Julio Cesar Ortega Iglesias, ** Tania Soledad Licona Rivera***}

\section{RESUMEN}

Antecedentes: La sibilancia es un trastorno muy frecuente en la infancia que contabiliza alta demanda de consulta médica y atención en los servicios de emergencia. Tiene relativamente altas tasas de hospitalización y está relacionado con la mortalidad infantil inducida por infecciones respiratorias. Objetivo: Establecer las características clínicas y epidemiológicas de los lactantes hospitalizados en el Mario Catarino Rivas durante el período 15 de junio a 16 de septiembre del 2015 y determinar la utilidad del cuestionario Estudio Internacional de las Sibilancias en el Lactante (EISL) modificado aplicado a padres/responsables. Pacientes y Métodos: Estudio descriptivo transversal no experimental. Los padres de 100 niños con edades comprendidas entre 6 y 18 meses hospitalizados en la sala lactantes respondieron a través de una entrevista directa el cuestionario EISL modificado. Para las características de los lactantes, se utilizaron frecuencias, porcentajes y medidas de tendencia central, para valorar la utilidad del test, se calculó confiabilidad y validez total. Los datos se analizaron con SPSS versión 21.0 y Excel. Resultados: la prevalencia de sibilancias recurrentes encontrada fue del $31 \%(n=31)$; confiabilidad del cuestionario se valoró con el a cronba$\mathrm{ch}=0.841$ y KR-20=0.8333; validando el instrumento con validez de contenido: juicio de expertos 0.88 , validez de criterio: $r x y \geq 0.83$, validez de constructo: $\mathrm{KMO}=0.68$; la validez

\footnotetext{
* Médico residente de tercer año del posgrado de Pediatría. Universidad Nacional Autónoma de Honduras, Valle de Sula, UNAH-VS.

** Pediatra Neumólogo HNMCR.

*** Coordinadora investigación científica posgrado medicina EUCS UNAH-VS.

Dirigir correspondencia a: elizabethdrhn@hotmail.es

Recibido: 16 de noviembre 2015 Aprobado: 18 de febrero 2016
}

total fue de 0.79 Conclusiones: la prevalencia de sibilancias es más alta que lo reportado en la literatura para América Latina; el cuestionario acortado EISL es válido estadísticamente para ser aplicado en el Hospital Mario Catarino Rivas en Lactantes.

\section{PALABRAS CLAVE}

Cuestionarios, preescolar, Ruidos respiratorios.

\section{ABSTRACT}

Background: Wheezing is a very common disorder in childhood that recorded high demand for medical consultation and care in emergency services. It has relatively high rates of hospitalization and it is associated with infant mortality induced by respiratory infections. Objectives: To establish the clinical and epidemiological characteristics of infants hospitalized in the Mario Catarino Rivas Hospital (HNMCR) during the period June 15 to September 16, 2015 and to determine the usefulness of the International Study of Wheezing in Infancy (EISL) questionnaire. Patients and Methods: This is a not experimental cross-sectional study. Parents of 100 hospitalized children between 6 and 18 months answered through a direct interview the EISL modified questionnaire. To analyze the infants characteristics frequencies, percentages and central tendency measures were used. The reliability and overall validity was calculated to assess the usefulness of the test. Data were analyzed with the SPSS software version 21.0. Results: The prevalence of recurrent wheezing found was $31 \%(n=31)$; reliability of the questionnaire was assessed with Cronbach $a=0.841$ and KR-20 $=0.8333$; the instrument content validity: expert opinion 0.88 , criterion validity: $r x y \geq$ 0.83 , construct validity: $\mathrm{KMO}=0.68$; total validit 
y was 0.79 Conclusions: The prevalence of wheezing is higher than reported in the literature for Latin America; the shortened EISL questionnaire is statistically valid to be applied to the Mario Catarino Rivas Hospital Infants.

\section{KEY WORDS}

Questionnaires, preschool noise, Respiratory.

\section{INTRODUCCION}

Las sibilancias son un ruido adventicio continuo preferentemente espiratorio de tonalidad musical. El mecanismo que lo genera aún no se conoce con certeza, y aunque el movimiento de moco ha sido involucrado, la vibración de las paredes en una vía aérea con disminución en su luz parece jugar el papel más importante. ${ }^{(1,2)}$

La prevalencia de sibilancias en los lactantes se ha informado a nivel global de $45.2 \%$, aunque en América Latina es de $21.4 \%$ y en Europa de $15 \%$, con variedad de expresión de los factores relacionados en cada región, país o sociedad, un ejemplo es España con variaciones importantes. ${ }^{(3,4)}$ En Honduras, en la bibliografía revisada, no se encontró ninguna investigación al respecto.

A pesar del progreso medico actual, el lactante con episodios de sibilancias no ha sido estandarizado de forma clínico-epidemiológica, impidiendo que se caracterice correctamente la prevalencia y aspectos epidemiológicos de las sibilancias en la lactancia. Esfuerzo en curso actualmente gerenciado por el grupo voluntario del Estudio Internacional de Sibilancias en la Lactancia (EISL) que ha logrado estandarizar en parte de América Latina y Europa un cuestionario de 18 preguntas, que incluye los factores epidemiológicos y clínico-terapéuticos más importantes relacionados con el riesgo del lactante de sufrir sibilancias, de ser clasificado como sibilante recurrente y que una vez que esta línea de investigación haya consolidado dicha información a nivel global, empezará a generar estudios multicéntricos a nivel interna- cional para la predicción de asma utilizando los datos estandarizados obtenidos de la aplicación y validación de dicho cuestionario a nivel mundial en lactantes. ${ }^{(5,6)}$

El objetivo fue describir la prevalencia y otras características clínico-epidemiológicas de las sibilancias en el lactante atendido en el Hospital Mario Catarino Rivas (HNMCR) entre el 15 de Junio y el 15 de Septiembre del año 2015 y la utilidad del cuestionario Estudio Internacional de las Sibilancias en el Lactante (EISL) modificado aplicado a padres/responsables.

\section{PACIENTES Y METODOS}

Se realizó un estudio con enfoque cuantitativo, diseño no experimental transversal y de alcance descriptivo. El universo fueron 262 niños con edades entre 6-18 meses ingresados en sala de lactantes del Hospital "Mario Catarino Rivas" entre 15 de Junio y 15 de Septiembre 2015; se realizó un muestreo probabilístico sistemático de 100 pacientes. Los criterios de inclusión fueron: lactante con edad entre 6-18 meses hospitalizados en la sala de lactantes con sibilancias cuyos padres/responsables aceptaron ser incluidos en el estudio; excluyéndose de este la comorbilidad pulmonar (Tuberculosis, enfermedad congénito pulmonar, fibrosis quística, inmunodeficiencia), edades $<6$ meses $0>18$ meses y que sus padres no aceptaron ser incluidos.

Previo a la aplicación de los instrumentos se obtuvo permiso de la Institución, se procedió a solicitar los expedientes clínicos de todos los casos y el investigador entrevistó directamente al padre/responsable que estuvo dispuesto a participar. Debido a que se trata de una investigación no experimental, en la que no existe riesgo alguno para los participantes, es un estudio categoría I.

Se recolectó la información mediante el cuestionario EISL modificado, el cual consta de 20 preguntas epidemiológicas y 18 dicotómicas para valorar las características clínicas. Previa- 
mente se aplicó una prueba piloto y evaluación del contenido del instrumento mediante juicio de expertos.

Para la caracterización de lactante sibilante recurrente se tomó a los niños menores de 2 años de edad que haya presentado 3 episodios o más de cuadros respiratorios con sibilancias en el periodo de un año. Para su análisis se utilizaron frecuencias, porcentajes y medidas de tendencia central. De los 100 niños menores de 18 meses, 31 tuvieron 3 o más episodios de sibilancias en un año, que conformará la población del análisis inicial de este estudio.

Para la evaluación de la utilidad del cuestionario modificado EISL se establecieron las siguientes hipótesis: Hipótesis nula: El cuestionario modificado EISL no es válido estadísticamente para ser aplicado en el Hospital Mario Catarino Rivas en Lactantes. Hipótesis alterna: El cuestionario modificado de la EISL es válido estadísticamente para ser aplicado en el Hospital Mario Catarino Rivas en Lactantes. Para que dicho test pueda usarse, debe calcularse la confiabilidad y validez del instrumento.

La CONFIABILIDAD es el grado que la aplicación repetida de un instrumento de medición al mismo individuo u objeto producen resultados iguales. Existen diferentes fórmulas para calcular la confiabilidad de un instrumento entre ellas tenemos: 1. Medida de estabilidad (confiabilidad por test-retest), 2. Método de formas alternativas o paralelas, 3. Método de mitades partidas (spit-halves), 4. Medidas consistencia interna u homogeneidad: KR-20 (Ver figura No.1) que se utiliza para ítems dicotómicos como el instrumento de esta investigación, sin embargo, también se calculó alfa de Cronbach debido a que era necesario para obtener la validez de criterio y compararla con el alfa de Cronbach del EISL. ${ }^{(7,8)}$

La VALIDEZ DE UN TEST se refiere al grado en que un instrumento mide realmente la variable que pretende medir. Se calcula mediante la validez de contenido, de criterio y de constructo. La validez de contenido se refiere al grado en que un instrumento refleja un dominio específico de contenido de los que se mide (juicio de expertos). La validez de criterio (rxy) (Ver figura No. 2) establece la validez de un instrumento de medición al compararlo con algún criterio externo que pretende medir lo mismo. $^{(7)}$

La validez de constructo es la más importante sobre todo desde una perspectiva científica, aporta mayor evidencia sobre la validez general de un instrumento de medición. Un constructo es una variable medida que tiene lugar dentro una hipótesis, teoría o modelo teórico, se calcula mediante análisis factorial. ${ }^{(7,9)}$

Se calculó la medida de adecuación de la muestra KMO (kayser, Meyer y Olkin) que es un índice que toma valores entre 0 y 1 , que se utiliza para comparar las magnitudes de los coeficientes de correlación observados con las magnitudes de los coeficientes de correlación parcial de forma que, cuanto más pequeño sea su valor, mayor es el valor de los coeficientes de correlación parciales y por lo tanto menos valido es el instrumento. La interpretación de los resultados está dado por los siguientes valores:

$\mathrm{KMO} \geq 0,75=$ Bueno, $\mathrm{KMO} \geq 0,5=$ Aceptable, $\mathrm{KMO}<0,5=$ Inaceptable.

La prueba de esfericidad de Bartlett contrasta bajo la hipótesis de normalidad multivariante, si la matriz de correlación de las $p$ variables observadas ( $p$ R) es la identidad. Si el nivel crítico o de significancia es mayor que 0,05 no podremos rechazar la hipótesis nula de esfericidad y consecuentemente no podremos asegurar que el modelo factorial sea adecuado para explicar los datos. ${ }^{(9,10)}$

Se calculó el tamaño de muestra con el programa EpiDat3.1 (OPS-OMS/Xunta de Saúde, Galicia, España, 2006). Se utilizó el programa IBM-SPSS versión 21.0 (IBM-SPSS Inc. Cary, NC, 
EUA, 2012) para obtener las medidas de tendencia central, calcular alfa de cronbach y validez de constructo. Se utilizó el programa Excel para hacer tablas, gráficos y calcular el KR-20.

\section{RESULTADOS}

La prevalencia de lactantes sibilantes recurrentes encontrada fue de 31 (31\%) de los 100 pacientes que se les aplico el cuestionario EISL modificado.

De estos lactantes sibilantes recurrentes 17 (55\%) eran masculinos y 14 (45\%) femeninos. Encontrándose una media de edad de 10.1 meses; mediana de 9.0; moda: 8; desviación estándar 3.72 y rango 6-18. (Ver Gráfico No. 1)

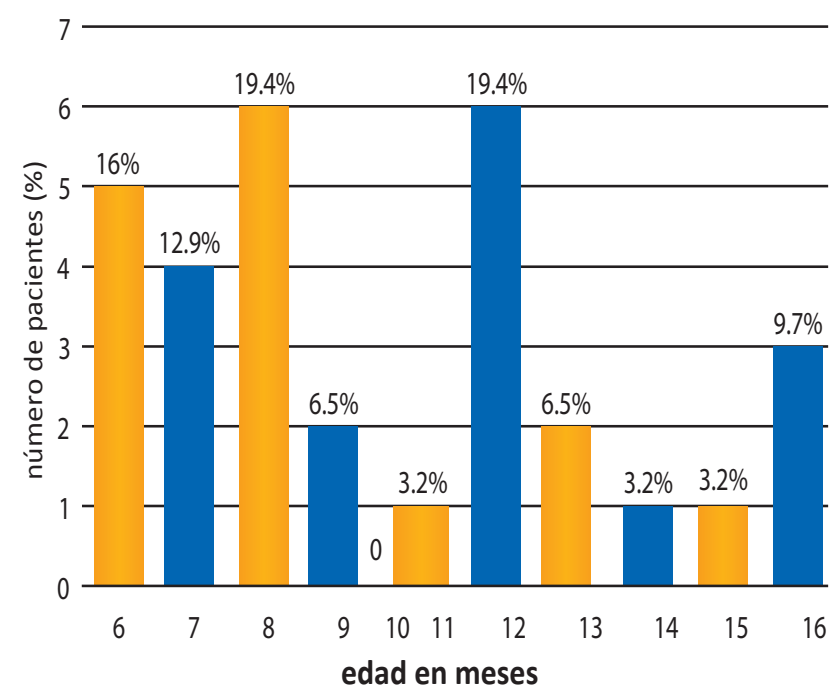

Gráfico No. 1: Distribución de los lactantes sibilantes recurrentes según la edad en meses.

Fuente: Estudio de Tesis Caracterización del Lactante Sibilante y Utilidad del Estudio Internacional de Sibilancias, Hospital Mario Catarino Rivas, 2015

Procediendo de un ambiente rural 15(48.4\%), urbano $10(32 \%)$ y urbano marginal 6(19\%). La escolaridad del cuidador analfabeta $2(6.5 \%)$, primaria incompleta $2(6.5 \%)$, primaria completa $14(45 \%)$, secundaria incompleta $8(25.8 \%)$, secundaria completa $3(9.7 \%)$ y universidad incompleta 2(6.5\%).
Se encontró que de los lactantes sibilantes recurrentes 29 (93.5\%) presentaron sibilancias o bronquiolitis en los primeros 12 meses de vida, 28 (90.3\%) presentaron sibilancias o bronquitis en los últimos 12 meses, 30(96.8\%) han utilizado tratamiento con B2 agonistas de acción corta inhalados o nebulizados, 14 (45.2\%) han utilizado tratamiento con corticoesterioides inhalados, 11 (35.5\%) tratamiento con esteroides orales y ninguno ha utilizado antileucotrienos.

La utilidad del cuestionario se determinó mediante: La confiabilidad (Ver figura No.1) con un KR-20 $=0.8333$ y a de cronbach $=0.841$; La validez del instrumento se realizó a través de: validez de contenido: juicio de expertos 0.88 , validez de criterio por el coeficiente de validez (rxy) (Ver figura No. 2) obteniendo $r x y=0.83$ y la validez de constructo se hizo utilizando el análisis multifactorial, donde para realizar el análisis de factores se eliminó la pregunta ¿algún médico diagnosticó a su bebe por tener asma? Porque presentaba una comunalidad $<0.4$ y hacía que la validez del instrumento fuera inaceptable.

$$
\begin{aligned}
& K R-20=(\mathrm{k} / \mathrm{k}-1)\left(\sigma^{2} \Sigma \mathrm{pq} / \sigma^{2}\right) \\
& K R-20=\left(\frac{18}{18-1}\right)\left(\frac{(15.6819)(0.7871)}{15.6819}\right) \\
& K R-20=(1.0588)\left(\frac{12.3432}{15.6819}\right) \\
& K R-20=(1.0588)(0.7871) \\
& K R-20=0.8333
\end{aligned}
$$

Figura No. 1: Cálculo de la confiabiliad del instrumento (Test de Kuder Richardson KR-20)

$\mathrm{k}=$ número de ítems del instrumento, $\mathrm{P}=$ proporción de respuestas correctas, $q=1-p, \sigma 2=$ varianza total del instrumento.

Fuente: Campo-Arias A, Oviedo H. Propiedades Psicométricas de una Escala: la Consistencia Interna.(8) 


$$
\begin{aligned}
& r x y=\sqrt{(r x x)(r y y)} \\
& r x y=\sqrt{(r x x)(r y y)} \\
& r x y=\sqrt{(0.84)(0.82)} \\
& r x y=0.83
\end{aligned}
$$

Figura No. 2: La fórmula para calcular Validez de criterio (rxy) del instrumento

$r x y=$ coeficiente de validez (correlación entre la prueba y el criterio), rxx= confiabilidad de la prueba, $r x y=$ confiabilidad de criterio.

Fuente: Hernandez Sampieri R, Fernández Collado C, Baptista L. Metodología de la investigación. 5ta ed. Mexico: McGrawHill; 2010.

Para determinar el grado de asociación entre las variables se utilizó medida de adecuación de la muestra KMO (Kayser, Meyer y Olkin) y la prueba de esfericidad de Bartlett.

(Ver tabla No. 1)

Tabla 1. KMO y prueba de Bartlett

\begin{tabular}{|l|l|}
\hline $\begin{array}{l}\text { Medida de adecuación muestral de } \\
\text { Kaiser-Meyer-Olkin }\end{array}$ &, 688 \\
Prueba de esfericidad de Barlett & \\
Chi-cuadrado aproximado & 817,924 \\
Gl & 210 \\
Sig. &, 000 \\
\hline
\end{tabular}

Fuente: Estudio de Tesis Caracterización del Lactante Sibilante y Utilidad del Estudio Internacional de Sibilancias, Hospital Mario Catarino Rivas, 2015

La validez total se refiere a la sumatoria de la validez de contenido (0.88), validez de criterio (0.83) y la validez de constructo (0.688). La validez total obtenida es de 0.79 .

\section{DISCUSION}

Las diferencias epidemiológicas entre países ó regiones respecto a la prevalencia de sibilancias son notorias; aunque se ha reportado prevalencia global de hasta $45.2 \%$, en Europa y Latinoamérica las cifras son de $15 \%$ y $21.4 \%$ respectivamente. Estudios españoles también reportan amplias diferencias entre regiones de dicho país, con características epidemiológicas particulares. ${ }^{(3,4)}$ Todo esto hace necesario generar la información local, ya que la internacional no es extrapolable y en Honduras no se dispone de dicha información; en este estudio la prevalencia de sibilancias en los lactantes hospitalizados estudiados fue $31 \%$, lo que es superior a las prevalencias generales para América Latina y Europa, aunque hay que hacer notar que es inferior a la estimación global.

La prevalencia de sibilancias encontrada fue mayor en los lactantes masculinos $55 \%$ que las niñas 45\%, hecho que concuerda con el estudio de Mallol en Brasil donde los resultados globales del EISL han mostrado una serie de factores de riesgo asociados a sibilancias en el primer año de vida, entre estos el sexo masculino aparecía como factor de riesgo de sibilancias recurrentes durante los primeros meses de vida. ${ }^{(5,13)}$ Esto podría estar relacionado con el hecho de que los niños tengan las vías aéreas más pequeñas en relación al tamaño pulmonar, lo que podría causar una mayor sensibilidad a irritantes $u$ otros agentes inhalados. $^{(11,12)}$

Los resultados de este estudio muestran que la edad en que se presentaron con mayor frecuencia las sibilancias recurrentes, es entre los 8 y 12 meses (19.4\% en cada grupo), además se encontró que $93.5 \%$ de los lactantes sibilantes recurrentes habían presentado sibilancias o bronquiolitis en los primeros 12 meses de vida, en concordancia con lo reportado en el estudio Dela Bianca sobre el inicio temprano de las sibilancias recurrentes ${ }^{(6,14)}$ y el de Chong Neto donde se encontró que 45.4\% sufrieron episodios de sibilancias durante los primeros 12 meses de vida, con promedio de edad entre $5.5+/-3.1^{(15)}$

La confiabilidad de esta prueba se hizo calculando el KR-20 $=0.8333$ demostrando un alto nivel de confianza. Además, para fines prácticos y poder calcular la validez de criterio, se 
calculó la confiabilidad con el alfa de cronbach $=0.841$ que demuestra un nivel de fiabilidad muy buena (intervalo entre 0.8-0.9) por lo que se da una buena aceptabilidad del instrumento. ${ }^{(8)}$ Estos resultados, están en concordancia con los datos obtenidos en el EISL realizado por de la Bianca en Brasil donde reportaron un alfa de cronbach $=0.823 .{ }^{(16)}$

Para calcular la validez total del instrumento fue necesario determinar la validez de criterio $(r x y)=$ 0.83 , la cual se realizó obteniendo la raíz cuadrada del alfa de cronbach de este estudio (0.841) multiplicada por la del criterio utilizado (0.823) que fue la del estudio de Dela Bianca en Brasil. ${ }^{(16)}$ Ambos instrumentos demostraron tener mediciones 0.8 por lo que la validez de criterio de este instrumento resultó tener alta correlación con el instrumento comparado. ${ }^{(7)} \mathrm{Sin}$ embargo, en la bibliografía consultada, no se encontró otro estudio similar donde hubiesen calculado la validez de criterio al EISL modificado.

Para calcular la validez de constructo, se computó el $\mathrm{KMO}=0,688$ manifestando que la validez del instrumento es aceptable (KMO> $0,5)$ con la prueba de esfericidad de Bartlett se obtuvo un valor de $\mathrm{X} 2$ alto $(817,92)$, con un nivel de significancia estadística < de 0.05 $(0.000)$, rechazando la hipótesis nula, indicando que si existen relaciones significativas entre las variables, confirmándose la validez aceptable del instrumento. ${ }^{(9)}$ Tampoco se encontraron estudios donde se hubiera calculado la validez de constructo al EISL modificado, siendo este un aporte de esta investigación.

Entre las fortalezas de este estudio están que es el primero que reporta la prevalencia de sibilancias en la población de lactantes en pacientes captados en un medio ambiente hospitalario que no se relaciona directamente con el ingreso de pacientes con patología del sistema respiratorio, sino más bien en una sala común, lo que permite extrapolar los hallazgos a todos los lactantes atendidos por esta institución de tercer nivel. Entre las limitantes están el tamaño de muestra, que para que sea posible extrapolar las conclusiones de este estudio a la población nacional de lactantes se necesita aumentar el tamaño de la muestra de acuerdo a las recomendaciones del grupo EISL.

En conclusión, la prevalencia de sibilancias recurrentes en lactantes hospitalizados en el Mario Catarino Rivas, es más alta que lo reportado en la literatura para América Latina. El cuestionario acortado de la EISL es válido estadísticamente para ser aplicado en lactantes del Hospital Mario Catarino Rivas.

Es recomendable extender el período del estudio hasta acumular muestras mayores de 1100 casos. Ampliar la implementación del estudio a otros centros de tercer nivel como el Hospital Escuela Universitario en Tegucigalpa y Hospital de Especialidades del Instituto Hondureño de Seguridad Social (IHSS) para estandarizar diagnóstico de sibilancias e implementar estudios de seguimiento de estos pacientes para determinar la relación entre los factores y preguntas de cuestionario acortado de EISL y riesgo de asma en la edad preescolar.

\section{AGRADECIMIENTOS}

Al Dr. Marvin Rodríguez Moreno, PhD por su valiosa colaboración en el análisis estadístico en la validez de criterio del instrumento. 


\section{BIBLIOGRAFÍA}

1. Coronel C, López A, González G, Cotarelo M. Problemas implicados en el estertor sibilante en niños menores de cinco años. Mex Pediatr. [Revista en internet] 2013 [citado 15 de mayo 2015]; 80(5): 185-190. Disponible en: http://www.medigraphic. com /pdfs/pediat/sp-2013/sp135c.pdf.

2. Pellegrini J, De Arriba S. Mesa Redonda: Problemática alergológica en pediatría. Sibilancias en el lactante. Bol Pediatr. [Revista en internet] 2014 [citado 15 de mayo 2015]; 54:72-7. Disponible en: https:// www.sccalp.org/documents/0000/2058/ BolPediatr2014_54_72_77.pdf.

3. Mallol J, Garcia-Marcos L, Sole D, Brand P, Group EISL. International prevalence of recurrent wheezing during the first year of life: variability, treatment patterns and use of health resources. Thorax. [Revista en internet] 2010 [citado 15 de mayo 2015]; 65(11):1004-1009. Disponible en: http:// thorax.bmj.com/content/65/11/1004.full. pdf.

4. Sanchez L, Akiko O, Mallol J, Solé D. Prevalence and clinical characteristics of wheezing in children in the first year of life, living in Cuiabá, Mato Grosso, Brazil. Rev. Paul. Pediatr. [Revista en Internet]. 2014 [citado 10 de junio 2015]; 32(4): 313-319. Disponible en: http://www.sciencedirect.com/sci ence/article/pii/S2359348215300658.

5. Mallol J, Garcia-Marcos L, Aguirre V, Martinez-Torres A, Perez-Fernandez V, Gallardo $A$, et al. The International Study of Wheezing in Infants: questionnaire validation. Int Arch Allergy Immunol. [Revista en internet] 2007 [citado 10 de junio 2015]; 144(1): 44-50. Disponible en: http://www. ncbi.nlm.nih.gov/pubmed/17505136.

6. Dela Bianca AC, Wandalsen GF, Mallol J,
Sole D. Prevalence and severity of wheezing in the first year of life. J Bras Pneumol. [Revista en internet] 2010 [citado 15 de mayo 2015]; 36(4): 402-9. Disponible en: http://www.ncbi.nlm.nih.gov/pubmed/ 20835585.

7. Hernandez Sampieri R, Fernández Collado C, Baptista L. Metodología de la investigación. 5ta ed. Mexico: McGraw-Hill; 2010.

8. Campo-Arias A, Oviedo H. Propiedades Psicométricas de una Escala: la Consistencia Interna. Salud pública. [Revista en internet] 2008[citado 10 de febrero 2016]; 10 (5): 831-839. Disponible en: http://www.reda lyc.org/articulo.oa?id=42210515.

9. Carvajal A, Centeno C, Watson R, Martínez M, Sanz Rubiales Á. ¿Cómo validar un instrumento de medida de la salud? Anales Sis San Navarra [revista en Internet]. 2011 [citado 12 febrero 2016]; 34(1): 63-72. Disponible en: http://scielo.isciii.es/scielo. php?script $=$ sci_arttext $\&$ pid $=$ S1 137-662 $72011000100007 \&$ Ing=es.

10. Fernández RF, Zapata ZC, Díaz VC, Taypicahuana JJL. Validación de un instrumento para medir la actitud hacia la donación de órganos en familiares de pacientes hospitalizados. Rev Cuerpo Médico HNAAA. [revista en Internet]. 2014 [citado 12 febrero 2016]; 7(1): 24-28 Disponible en: https:// www.google.hn/url?sa=t\&rct=j\&q=\&esrc= s\&source $=$ web\& $c d=5 \&$ ved $=0$ ahUKEwiX5 $4 \mathrm{fzrfHKAhVMbj} 4 \mathrm{KHbgRAsMQFgg} 9 \mathrm{M}$ AQ\&url=http\%3A\%2F\%2Fdialnet.uniri oja.es\%2 Fdescarga\%2 Farticulo\% 2 F4755890.pdf\&usg=AFQjCNG5dYYTbDrq MU2F408efOOKI9zq3w.

11. Puig C, Fríguls B, Gómez M, García-Algar O, Sunyer J, Vall O. Relación entre las infecciones respiratorias de vías bajas durante el 
primer año de vida y el desarrollo de asma y sibilancias en niños. Arch Bronconeumol. [Revista en Internet]. 2010 [citado 12 de junio 2015]; 46(10):514-521. Disponible en: http://www.archbronconeumol.org/es/re lacion-entre-las-infecciones-respiratorias/ articulo/S0300289610001997/.

12. Medeiros D, Silva A, Rizzo J, Sarinho E, Mallol J, Sole D. Prevalence of wheezing and associated risk factors among infants in Recife, Pernambuco State, Brazil. Cad Saude Pública. [Revista en Internet]. 2011[citado 10 de junio 2015]; 27(8):1551-9. Disponible en: http://www.ncbi.nlm.nih.gov/pubmed/ 21877003.

13. Bozaykut A, Paketci A, Gonul R, Paketcic C. Evaluation of Risk Factors for Recurrent Wheezing Episodes. J Clin Med Res. [Revista en Internet]. 2013 [citado 10 de junio 2015]; 5(5): 395-400. Disponible en: http://www. ncbi.nlm.nih.gov/pmc/articles/PMC374 8665/.
14. Moraes L, Takano O, Mallol J, Sole D. Risk factors associated with wheezing in infants. J Pediatr (Rio J). [Revista en Internet]. 2013 [citado 10 de junio 2015]; 89(6):559-566. Disponible en: http://www.sciencedirect. com/science/article/pii/S2255553613001 274.

15. Chong Neto HJ, Rosario NA, Grasselli EA, Silva FC, Bojarski Lde F, Rosario CS, et al. Recurrent wheezing in infants: epidemiological changes. J Pediatr (Rio J). [Revista en Internet] 2011[citado 15 de mayo 2015]; 87(6):547-50. Disponible en: http://www.n cbi.nlm.nih.gov/pubmed/21850363.

16. A. C. Dela Bianca, G. F. Wandalsen, K. Miyagi, L. Camargo, D. Cezarin, J. Mallol, D. Solé. International Study of Wheezing in Infants (EISL): validation of written questionnaire for children aged below 3 years. J Investig Allergol Clin Immunol. [Revista en Internet] 2009[citado 15 de mayo 2015]; 19 (1): 3542. Disponible en: http://www.ncbi.nlm. nih.gov/pubmed/19274927. 